\title{
Oedema of the ileum: a possible manifestation of food allergy
}

The mechanisms whereby adverse reactions to food cause gastrointestinal symptoms are poorly understood. In most cases of food allergy there are no reliable radiological, histological, or immunological abnormalities on which to base the diagnosis, which depends on subjective improvement of symptoms on withdrawal of the offending agent with or without a subsequent positive reaction to challenge. We report a case of presumed food attergy with clear cut reversible radiological changes in the mucosa of the small bowel.

\section{Case report}

A 39 year old woman developed recurrent episodes of giant urticaria with no obvious precipitating cause. Four weeks later she began to suffer episodes of severe colicky upper umbilical pain, during which she was unable to eat or drink; there was no vomiting. Episodes lasted for up to 24 hours and recurred after about eight days. The attacks of pain increased in severity until the time of investigation, five months after onset. She had had a similar, less severe episode of pain five years previously, which had settled spontaneously. There was no family or personal history of atopy, and she was not taking any drugs.

On examination she had giant urticaria affecting the trunk and both arms and precipitated when a normal diet was reintroduced. Even if an antigen is correctly identified it is not clear for how long it should be avoided.

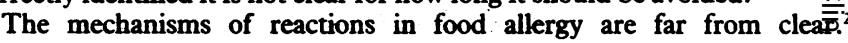
Presumably a food allergen cross links specific IgE in the mucosa of the smDD bowel, triggering a type I allergic reaction and causing abnormal permeability ${ }^{3}$ that allows systemic absorption of the same, or different, antigent, which then trigger the urticarial reaction in the skin. The response to histamine antagonists supports this hypothesis. Non-allergic reactio however, can produce a similar sequence of events, in which mast cells afte degranulated by mechanisms that do not rely on the immune system. The antigenic load probably diminishes down the small intestine because 의 digestion and absorption, and the distribution of mucosal mast cells and $\mathrm{I} F$ plasma cells remains uniform throughout the intestine, ${ }^{5}$ so it is surprisiong that the ileum was affected in this case and not the proximal small bowet. Finally, other possible causes of mucosal oedema, such as eosinophinc gastroenteritis or an allergic reaction to a parasitic infection, were mut definitely excluded.

The initial investigations in this patient yielded entirely normal results b出 were performed when she was free of symptoms. The abnormal result of the barium follow through examination during an attack of pain emphasises $\overline{3}$
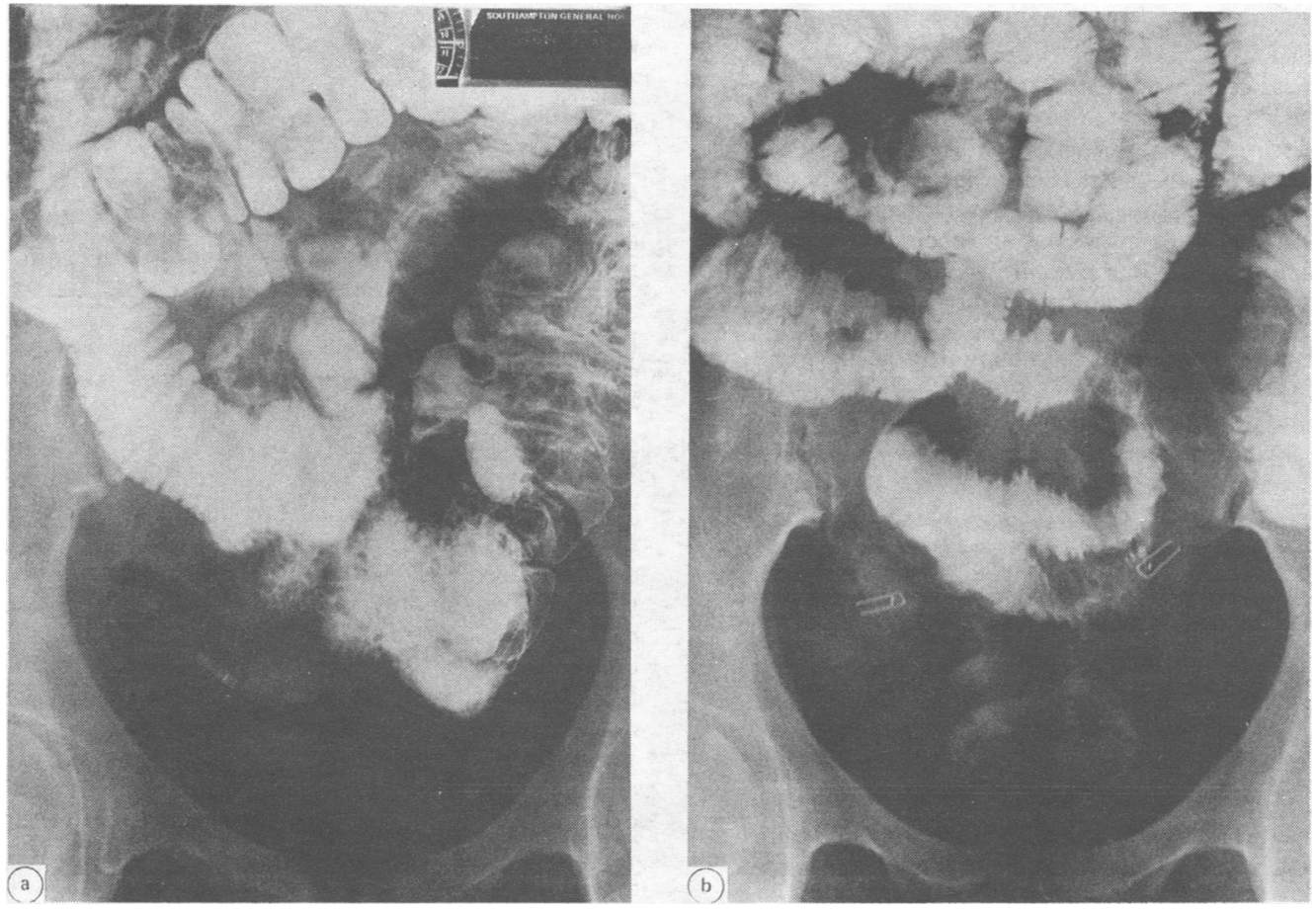

$x$ Ray films of terminal ileum obtained by barium follow through examination: $(a)$ when patient free of symptoms, showing normal mucosa, and $(b)$ during attack of abdominal pain, showing severe mucosal oedema.

legs. Abdominal examination yielded normal results. The following were normal: full blood count (4\% eosinophils); immunoglobulin and complement concentrations; $\mathrm{C}_{1}$ esterase inhibitor concentrations and activity; serum amylase activity; and results of autoantibody screening, intradermal skin tests for common allergens, abdominal ultrasonography, and barium follow through examination (figure $(a)$ ). Blood tests gave normal results seven days later during a typical attack of pain, but barium follow through examination (figure $(b)$ ) showed severe mucosal oedema affecting the terminal ileum.

Treatment included a standard oligoallergenic diet that avoided all additives, colourings, and preservatives, ${ }^{1}$ but her symptoms persisted. Terfenadine $180 \mathrm{mg}$ twice daily led to a reduction and later resolution of her abdominal pain, and cimetidine $\mathbf{4 0 0} \mathrm{mg}$ twice daily led to improvement of her rash. A normal diet was restarted without recurrence of her symptoms.

\section{Comment}

This case illustrates many of the problems associated with diagnosing food allergy. It can be extremely difficult to identify the antigenic substances in a diet containing many thousands of potential antigens, and in this case symptoms persisted during a diet that was free of antigens and were not importance of performing investigations in suspected food allergy wh patients are symptomatic, particularly if symptoms are episodic.

1 Francis DEM. Dietary management of food allergy. In: Challacombe S, Brostoff J, eds. Proceed in of the first food allergy workshop. Oxford: Medical Education Services Lid, 1980:85-94.

2 Wright R, Robertson DAF. Mechanisms of damage. Immunologically mediated-local distant. In: Brostoff J, Challacombe S, eds. Food allergy. Eastbourne: Baillière Tindei, 1986:237-47.

3 Perdue MH, Chung M, Grantgall D. Effect of intestinal anaphylaxis on gut function in the Tat. Gastroenterology 1984;86:391-7.

4 Moneret-Vautrin DA. False food allergies: non-specific reactions to foodstuffs. In: Lessof $M$, Clinical reactions to food. Chichester: John Wiley, 1983.

5 Befus AD, Pearce FL, Gauldie J, et al. Mucosal mast cells. I. Isolation and functiona characteristics of rat intestinal mast cells. $\mathcal{F}$ Immunol 1982;128:2475-80.

(Accepted 8 October 1986)

Professorial Medical Unit, Southampton General Hospital, Southampton SO9 4XY

DUNCAN ROBERTSON, MD, MRCP, lecturer in medicine RALPH WRIGHT, DPHIL, FRCP, professor of medicine

Correspondence to: Dr Robertson. . 